\title{
Atlas dynamique des zones inondables : outil operationnel d'aide à la décision
}

\author{
Bertrand JACOPIN ${ }^{(1)}$, Emilie ANDRIES, Yohann BEURENO(1), Fabienne MERCIER ${ }^{(1)}$ \\ Pierre-Yves VALANTIN ${ }^{(2)}$, Yann LABORDA ${ }^{(2)}$
}

(I)SMAVD, Syndicat Mixte d'Aménagement de la Vallée de la Durance - e-mail: contact@smavd.org

${ }^{(2)}$ SPCGD DREAL, Service de Prévision des Crues du Grand Delta - e-mail: yann.laborda@developpement-durable.gouv.fr

\begin{abstract}
RÉSUMÉ. - Le Syndicat Mixte d'Aménagement de la Vallée de la Durance (SMAVD) s'attache depuis de nombreuses années à compléter les portés à connaissances règlementaires par des études hydrauliques spécifiques. Pour cela, le SMAVD construit et exploite des modèles numériques Telemac 2D, qui, fréquemment mis à jour, reflètent fidèlement les mécaniques complexes d'inondation du secteur. Pour répondre aux besoins de ses communes membres et après un audit des acteurs de la prévention des risques, il est apparu prioritaire au SMAVD de développer un outil permettant de se préparer aux inondations, de transformer les prévisions de débits en prévision de zones inondables potentielles (ZIP). Sur un premier tronçon de rivière équipé de très nombreux ouvrages anciens peu fiables, les hydrauliciens du SMAVD ont élaboré une méthodologie de modélisation du cours d'eau permettant de représenter les conséquences aléatoires liées à la tenue ou à la rupture des ouvrages. Une gamme étendue de débits de débits de crue a été modélisée. La représentation graphique des résultats a été mise au point dans le cadre d'une large concertation : en étroite collaboration avec les représentants de la commune pilote de Villelaure (84) puis avec l'ensemble des utilisateurs identifiés. L'Atlas Dynamique des Zones Inondables (AZI) de la Durance constitue le document pivot des plans communaux de sauvegarde, un outil de mesure des conséquences des potentielles ruptures d'ouvrages, le chainon manquant entre prévision de débit et prévision d'inondation mais également une aide précieuse lors des projections opérationnelles des services de secours.

Première action mise en œuvre dans la Stratégie Locale de Gestion du Risque Inondation (SLGRI) de la Durance, le projet AZI se déploie en régie interne au sein du SMAVD. Il couvre plus de $100 \mathrm{~km}$ de rivière. Il contribue au projet national Viginonde en mettant à disposition du Service de Prévision des Crues les différentes couches SIG des Zones Inondables Potentielles. L'outil sera par ailleurs largement partagé, avec entre autres les gestionnaires routiers, les communes et EPCI, les SDIS...
\end{abstract}

Mots-clés : Atlas Zone Inondable, Durance, Carte, Modélisation

\section{Dynamic atlas of flood-risk areas, operational tool of decision-making support}

\begin{abstract}
The SMAVD takes care for several years to the statutory knowledge with hydraulic specific studies. For that purpose, the SMAVD builds and exploits Telemac2D models, which, frequently updated, shows faithfully the complex flood mechanics on the area. To meet the needs of its municipality member and after having heard the main risk prevention actors, it seemed priority for the SMAVD to develop a tool allowing to get ready in front of floods, to transform the flow forecasts in anticipation of potential flood-risk areas and to produce useful information for an operational projection for emergency services.

On a first section of river which is equipped with a lot of unreliable old little works, the SMAVD hydraulicians developed a modelling methodology of the Durance allowing to represent the unpredictable consequences depending on the holding or the break of the hydraulic works. A vast range of flood flows was modelled. The finalization of the graphical representation of the results took place on a wide dialogue in close collaboration with the delegates of Villelaure municipality (84) then with all the identified users. The Durance Dynamic Atlas of Flood-risk areas is the centerpiece of the municipal protection plans set up, the measurement tool for the hydraulic works breaks consequences, the missing link between flow forecast and flood forecast but also a precious help during the emergency services operations.

The AZI project is built and realized in an internal SMAVD management. It's the fisrt action of the Durance SLGRI plan that was realized. It covers more than $100 \mathrm{~km}$ of the river. It contributes to the national project Viginonde by giving to the SPC Department the various SIG layers of the Potential Flood-risk areas. Moreover, the tool will be widely shared, with for example the road administrators, the municipalities and the public intermunicipal cooperation institute, the fire and rescue service...
\end{abstract}

Key-words: Dynamic atlas of flood-risk areas, Durance, Map, Model

\section{INTRODUCTION}

\section{I.1. La Durance}

La Durance prend naissance au col de Montgenèvre et se jette dans le Rhône $305 \mathrm{~km}$ plus loin. Son bassin versant représente une superficie d'environ $14280 \mathrm{~km}^{2}$, soit la moitié de la superficie de la Région PACA. Rivière alpine en pays méditerranéen, elle a toujours apporté ses hautes eaux de printemps et de début de l'été (fonte des neiges) alors que l'influence méditerranéenne l'emporte nettement dans la formation des crues dont les plus fortes ont généralement lieu à l'automne. 
Le caractère exceptionnel des crues de la Durance est dû à la puissance de la rivière (jusqu'à $5000 \mathrm{~m}^{3} / \mathrm{s}$ sur des pentes de 3 à 4\%o), à sa mobilité exceptionnelle (jusqu'à $1 \mathrm{~km}$ de largeur) qui permet une très grande extension des débordements. Les crues de 1994 ont rappelé brutalement la réalité du risque de débordements de la Durance malgré les grands réservoirs de Serre-Ponçon et du Verdon. Les très nombreux ouvrages en remblais qui jalonnent la vallée présentent souvent un niveau de protection incertain. De vastes zones sont protégées par des systèmes de protection par digues et épis, parfois complexes, voire anarchiques, dont le comportement en crue est imprévisible et peut parfois s'avérer brutal.

\section{I.2. Une rivière mobile, une organisation agile}

Suite aux crues de 1994 qui ont mis en évidence l'inadaptation du réseau dense et parfois anarchique des ouvrages en remblai dans la plaine de la Durance, un important programme de rénovation et de réorganisation des systèmes de protection a été engagé par le SMAVD afin d'offrir une protection adaptée aux enjeux de chaque secteur. Cette démarche a été conduite à la fois dans le souci d'identifier une ligne de défense compatible avec l'occupation de la plaine mais également dans l'objectif d'une amélioration de la capacité d'écoulement du lit. Le dimensionnement précis des ouvrages hydrauliques à reprendre dans le cadre de cette réorganisation nécessitait de nombreuses études hydrauliques sur une partie importante du territoire. Considérant le nécessaire travail de mise à jour imposé par le caractère très évolutif du lit de la Durance ainsi que des autres nécessités en matière de modélisation (études et suivis morphologiques notamment), le SMAVD a fait le choix d'internaliser ces prestations et de se doter d'un service spécialisé dès 2006.

Les premières modélisations ont rapidement montré que cette stratégie originale permettait la mise en ouvre d'un outil de dimensionnement puissant permettant d'affiner les projets et d'alimenter à la demande le service de maîtrise d'œuvre du syndicat. Au-delà, les mêmes outils ont facilité et structuré le suivi intégré des évolutions morphologiques du lit mineur comme des aménagements en lit majeur par le biais de mises à jour régulières et ont mis à disposition des informations cruciales quant à la conduite à tenir en période de crue (notamment lors de la crue de mai 2008).

Depuis lors le SMAVD construit, exploite et met à jour une série de modèles bidimensionnels de représentation des écoulements de la Durance en crue (Telemac 2D). La Durance est ainsi modélisée par tronçons sur une grande partie de son linéaire. Chaque modèle est généralement actualisé tous les quatre ans. Les mises à jour sont opérées en cas de modifications substantielles du lit mineur (après les crues les plus morphogènes) ou du lit majeur (après restructuration des ouvrages d'un système d'endiguement par exemple). Les résultats des calculs permettent de rendre compte, entre autres indicateurs :

- De l'évolution des lignes d'eau en crue dans le lit de la Durance et au droit des ouvrages de protection.

- De l'étendue des zones inondables, en fonction l'évolution morphologique et des aménagements de la vallée.

\section{I.3. Un besoin exprimé par les acteurs de terrain}

Les très nombreuses modélisations menées par le SMAVD dans le cadre de projets de restructuration des anciens systèmes d'endiguement confrontées aux cartographies règlementaires (PPRi) et mises en perspectives par les crues récentes ont très rapidement laissé apparaitre que :
- Le comportement des différents ouvrages en remblais est surdéterminant des mécaniques d'inondation et des zones inondées potentielles.

- Les Maires comme les services de secours sont souvent désemparés au moment de préparer ou gérer la crise inondation du fait de la complexité des mécaniques d'inondation en jeu.

- Les cartographies règlementaires se concentrent, par nature et lorsqu'elles existent, sur l'aléa de référence (Q100) et sont centrées sur les hypothèses les plus sécuritaires en vue d'être prises en compte dans les documents d'urbanisme.

Après un audit des acteurs de la gestion de crise et de la prévention des inondations, le constat suivant a été établit : le Maire, le commandant de Gendarmerie, le Préfet, le capitaine des pompiers, le gestionnaire du réseau de voirie départemental expriment tous le besoin de disposer de la description (dynamique) des zones du territoire menacées par un risque tangible d'inondation. L'expression de ce besoin traduit la volonté des différentes parties prenantes de :

- Se préparer en amont des crises sur des scénarios réalistes et non pas exclusivement extrêmes,

- Se projeter de manière opérationnelle en anticipant au maximum les inondations à venir,

- Evaluer les évolutions possibles des conséquences des crues pendant leur déroulé,

- De transformer les prévisions de débits lorsqu'elles existent en prévision de zones inondables potentielles (ZIP).

\section{CO CONSTRUCTION DU PREMIER ATLAS DYNAMIQUE DES ZONES INONDABLES}

\section{II.1. La prise en considération des ouvrages anciens}

En 2015, sur un premier tronçon de rivière d'environ $17 \mathrm{~km}$ équipé, doté de très nombreux ouvrages anciens et peu fiables, les hydrauliciens du SMAVD ont dans un premier temps élaboré une méthodologie de modélisation du cours d'eau permettant de représenter les conséquences aléatoires liées à la tenue ou à la rupture des ouvrages. En rive gauche, ce tronçon comprend les territoires communaux de la plaine alluviale de la Durance de Meyrargues, Le Puy-Sainte-Réparade, Saint Estève Janson et La Roque d'Anthéron. En rive droite, les communes de Pertuis, Villelaure, Cadenet, Puyvert et Lauris sont concernées.

Le modèle a été élaboré en vue de simuler la propagation d'hydrogrammes de crue en fonction du comportement des ouvrages et notamment de leurs conditions de ruptures selon trois scénarios :

1. Le scénario 1 «sans ruptures »: aucun ouvrage ne rompt. Cette configuration d'écoulement permet de comprendre la mécanique d'inondation de la plaine et le rôle des ouvrages structurants. Ce scénario peut ne pas être représentatif du comportement réel du réseau d'ouvrages pour les plus forts débits, notamment lorsque des surverses interviennent.

2. Le scénario 2 « ruptures probables »: approche tenant compte du comportement aléatoire des ouvrages existants. Pour cela, des conditions de rupture ont été appliquées sur la plupart des ouvrages. Une inspection méthodique des ouvrages a été réalisée afin de qualifier leur sensibilité à la surverse d'une part et à l'érosion interne d'autre part. Pour chacun de ces deux paramètres, trois niveaux de sensibilité à la rupture ont été retenus (cf. Table 1). Cette caractérisation des ouvrages est associée à la détermination de valeurs seuils pour la hauteur de surverse maximale que peut supporter l'ouvrage avant la rupture. Concernant la tolérance maximale des 


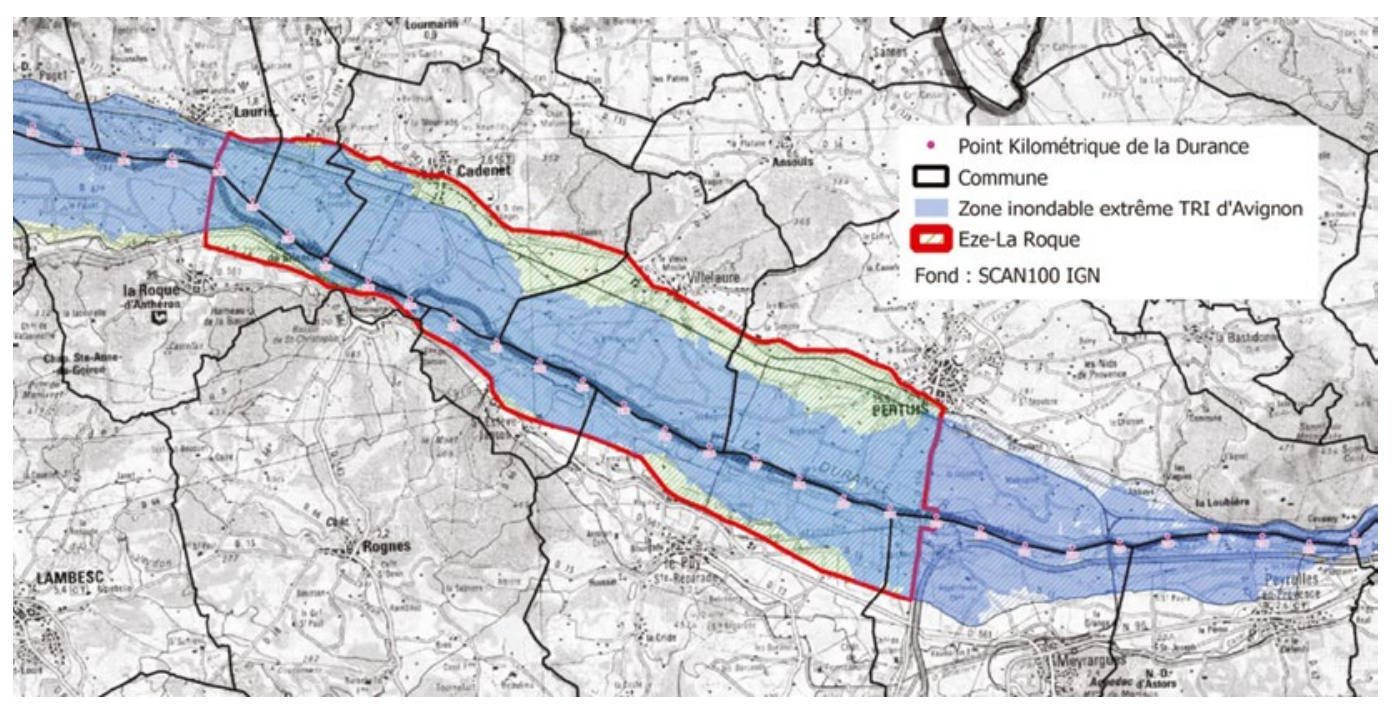

Figure 1 : Emprise du modèle numérique, sur fond de IGN Scan100.

\begin{tabular}{|c|c|c|c|c|}
\hline $\begin{array}{c}\text { Niveau de } \\
\text { sensibilité } \\
\text { de l'ouvrage }\end{array}$ & $\begin{array}{c}\text { Sensibilité à la } \\
\text { surverse }\end{array}$ & $\begin{array}{c}\text { Hauteur limite de } \\
\text { surverse }\end{array}$ & $\begin{array}{c}\text { Sensibilité à } \\
\text { l'érosion interne }\end{array}$ & $\begin{array}{c}\text { Gradient } \\
\text { limite }\end{array}$ \\
\hline Nul & S0 & - & E0 & \\
\hline Faible & S1 & $50 \mathrm{~cm}$ & E1 & 0.3 \\
\hline Fort & S2 & $10 \mathrm{~cm}$ & E2 & 0.2 \\
\hline
\end{tabular}

Table 1 : Caractérisation des ouvrages aux probabilités de ruptures par surverse et par érosion interne.

ouvrages à l'érosion interne, un gradient amont-aval maximal est défini. Il s'agit du rapport de la différence de cotes des surfaces libres amont-aval sur la largeur de l'ouvrage. Le niveau de sensibilité est estimé en fonction des observations suivantes : présence de végétation arborée, d'ouvrages traversant, de terriers, de revêtement de talus, nature et qualité du remblai quand cela est visible (limons, graves).

3. Le scénario 3 «emprise pessimiste» : correspond aux combinaisons de rupture/non rupture les plus défavorables. Elle correspond généralement à la rupture des ouvrages longitudinaux et transversaux amont favorisant les entrées d'eau, et à la non rupture des ouvrages transversaux aval maximisant les niveaux d'eau. Ce dernier scénario, donne une emprise globale, qui résulte de la concaténation d'une quinzaine de scénarios de rupture / non ruptures sur le tronçon modélisé.

\section{II.2. Depuis les premiers débordements jusqu'à la crue centennale}

Pour les trois scénarios de comportements combinés des ouvrages, les simulations numériques ont été réalisées à des débits de pointe de $1500,2000,2500,3000,4000$ et $5000 \mathrm{~m}^{3} / \mathrm{s}$ afin de traduire la mécanique d'inondation de manière quasiment continue depuis le « plein bord » jusqu'à l'aléa PPR.

Un hydrogramme simplifié, prenant en compte la forme générale de la crue de janvier 1994, a servi de base pour créer par homothétie les différents hydrogrammes appliqués au modèle. On retiendra que la forme des hydrogrammes des principales crues connues en Durance : octobre 1882, octobre et novembre 1886, janvier et novembre 1994, est relativement similaire à celui de la crue de 1994, avec un temps de montée de l'ordre de 15 à $20 \mathrm{~h}$.

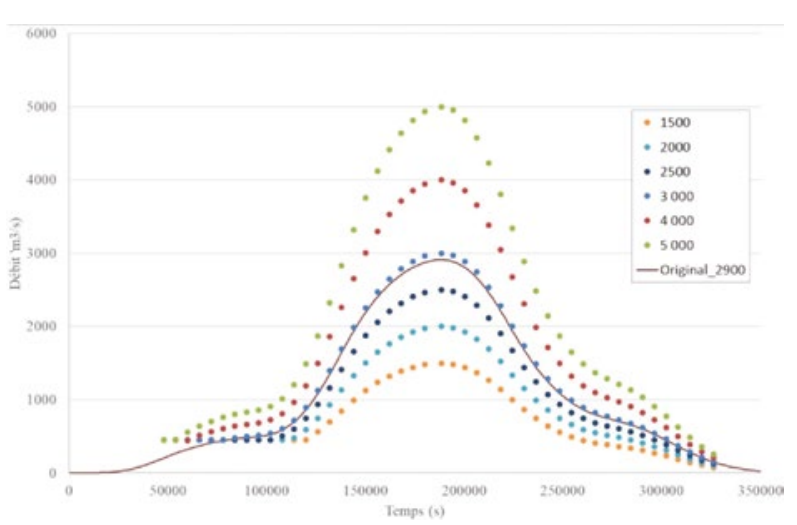

Figure 2 : Hydrogrammes des crues modélisées.

\section{II.3. Dans un cadre de mise à jour continue}

La crue de novembre 2016 et l'analyse en détail des résultats du modèle initial ont conduit à identifier quelques comportements inappropriés de l'outil (ruptures n'ayant pas lieu d'être) et ont conduit à reconsidérer une partie des critères. Les conditions de ruptures sur les ouvrages qui ont été mises en œuvre in fine sont les suivantes :

- Type 1: rupture par surverse $=>$ hauteur d'eau sur la crête $>10 \mathrm{~cm}$ et chute aval $>50 \mathrm{~cm}$

- Type 2 : rupture par surverse $=>$ hauteur d'eau sur la crête $>50 \mathrm{~cm}$ et chute aval $>100 \mathrm{~cm}$

- Type 3 : rupture par surverse $\Rightarrow>$ hauteur d'eau sur la crête $>10 \mathrm{~cm}$ et chute aval $>50 \mathrm{~cm} \mathrm{OU}$ rupture par gra- 
dient $=>$ hauteur d'eau $>50 \mathrm{~cm}$ et différence de côte maximale amont/aval $>20 \mathrm{~cm}$ ou $30 \%$ de la largeur ouvrage (selon classes de sensibilité de l'ouvrage) et $40 \%$ de la hauteur ouvrage (le plus petit des côtés si l'ouvrage est asymétrique), avec une hauteur d'eau minimale de $50 \mathrm{~cm}$,

- Type 4 : rupture par surverse $=>$ hauteur d'eau sur la crête $>50 \mathrm{~cm}$ et chute aval $>100 \mathrm{~cm} \mathrm{OU}$ rupture par gradient $=>$ hauteur d'eau $>50 \mathrm{~cm}$ et différence de côte maximale amont/ aval $>(20$ ou $30 \%$ de la largeur ouvrage et $40 \%$ de la hauteur ouvrage (le plus petit des côtés si l'ouvrage est asymétrique), avec une hauteur d'eau minimale de $50 \mathrm{~cm}$ ).

L'analyse des résultats obtenus est satisfaisante, toutes les ruptures ont été étudiées une à une et validée. Le fortran ainsi défini présente une bonne robustesse et montre une bonne pertinence des ruptures obtenues, dans tous les cas de figure (hauteur et largeur de l'ouvrage, conditions topo amont/aval, hauteurs d'eau...).

\section{II.4. La commune de Villelaure comme « modèle "}

Le volontarisme de l'équipe municipale, la bonne appréhension par le Maire des enjeux de préparation ou de gestion de la crise inondation ainsi que le programme de restructuration des ouvrages à l'étude, faisait de la commune de Villelaure le territoire idéal pour le développement de l'outil cartographique. Le groupe de travail constitué autour de Jean-Louis Robert (Maire) et Michel Crest (Adjoint délégué au SMAVD notamment) a permis d'aboutir à :

- Une cartographie grand public : la complexité des scénarios de tenues / ruptures des ouvrages doit se traduire par une représentation cartographique simple.

- Des formats de sortie adaptés à la préparation de la crise inondation (formats numériques et papiers classiques) : A3 paysage, PDF ou papier, données brutes *.SHP et mises en page QGIS

- Un outil de la gestion de crise permettant d'être lu sur le terrain en condition difficile : impression sur support hydrofuge (papier synthétique), brochage type Wir'o sur grand côté (haut).

La représentation graphique des résultats a été finalisée en prenant en considération les retours successifs des contributeurs de la SLGRI Durance et plus particulièrement des SDIS 13 et 84, de la Direction des Routes du Conseil Départemental de Vaucluse, de la DREAL PACA et du SPC Grand Delta.

\section{UN OUTIL DEPLOYÉ À GRANDE ÉCHELLE}

\section{III.1. Principes de déploiement}

Le service SIG du SMAVD, avec l'appui de Sophie Rommens (La Boite@ com'), a travaillé à un calepinage régulier de planches cartographiques au 1/15000 réparties le long de l'axe du cours d'eau. En anticipant la diversité des utilisateurs finaux (et donc de leur territoire), la numérotation des planches s'est basée sur la position de la carte le long de l'axe (PK). Chaque atlas peut donc couvrir un linéaire différent de Durance et respecter les mêmes concepts d'assemblage :

- Page de garde,

- Notice explicative et recommandations prudentielles,

- Plan d'assemblage,

- Planches cartographiques se succédant de l'aval vers l'amont et par débits croissants.

Chaque planche étant « autoporteuse », la mise à jour sera réalisée par substitution des planches concernées.

\section{III.2. Emprise et acteurs identifiés}

Les modélisations sont déployées en vue de la constitution de 80 planches cartographiques permettant de couvrir l'ensemble de la vallée de la commune de l'Escale jusqu'au confluent du Rhône soit plus de $150 \mathrm{~km}$. Sur ce périmètre, les atlas ont vocation à être mis à disposition : des communes (42), des EPCI (8), des trois Départements concernés, de la Région PACA, des grands gestionnaires de réseaux (RTE, ERDF, GDF, Vinci, SNCF...), des SDIS concernés et des services de l'Etat (DDT, Préfectures, SPC).

\section{III.3. Un partage sur la chaîne d'expertise et de décision}

L'atlas dynamique des zones inondables a vocation à devenir le référentiel commun des multiples acteurs de la gestion de crise inondation du territoire. En assurant la mise à disposition d'informations exhaustives et unifiées sur le comportement de la plaine en période de crue, l'outil permet d'une part une acculturation aux risques liés aux inondations et constitue d'autre part un pivot en matière de coordination en temps de crise.
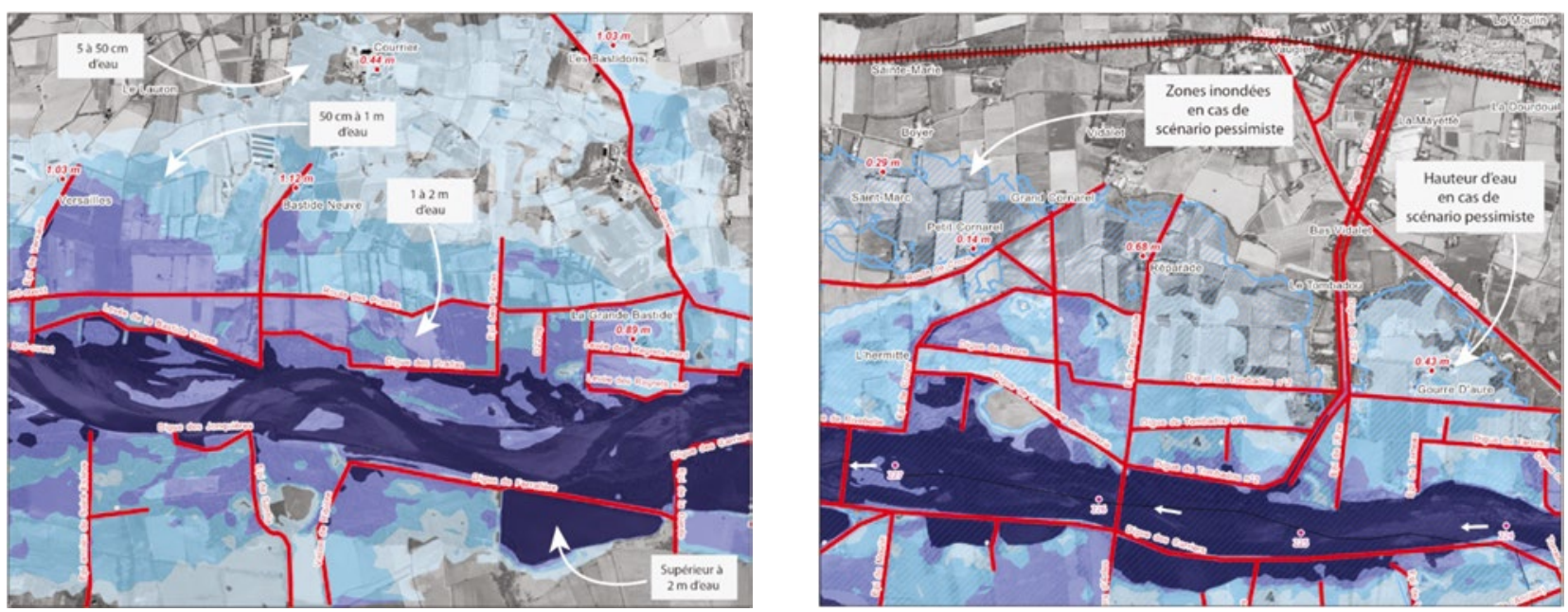

Figure 3 et 4 : Représentations dans un scénario " probable » et dans le scénario " pessimiste ». 


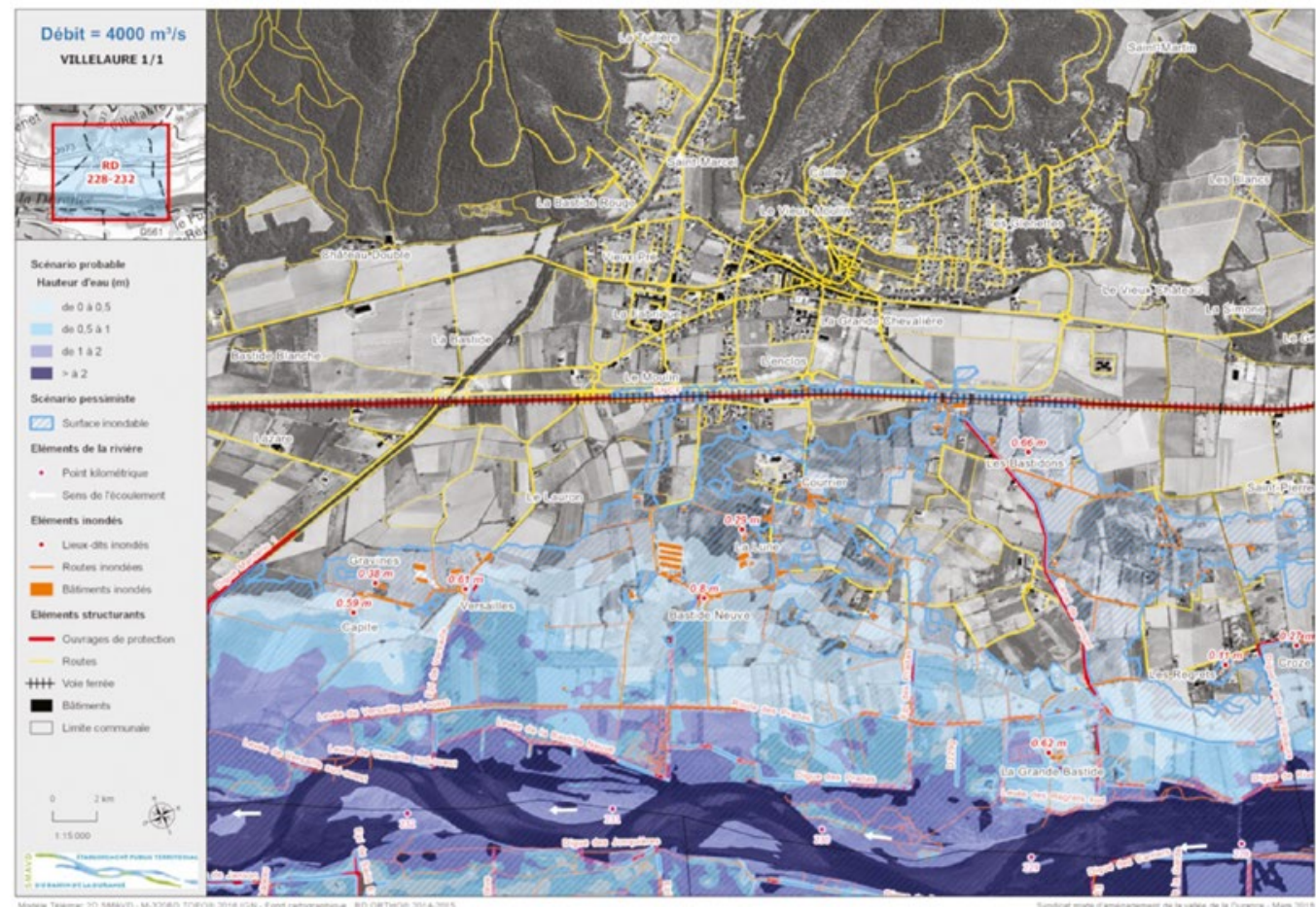

Figure 5 : Exemple de planche cartographique mise en page.

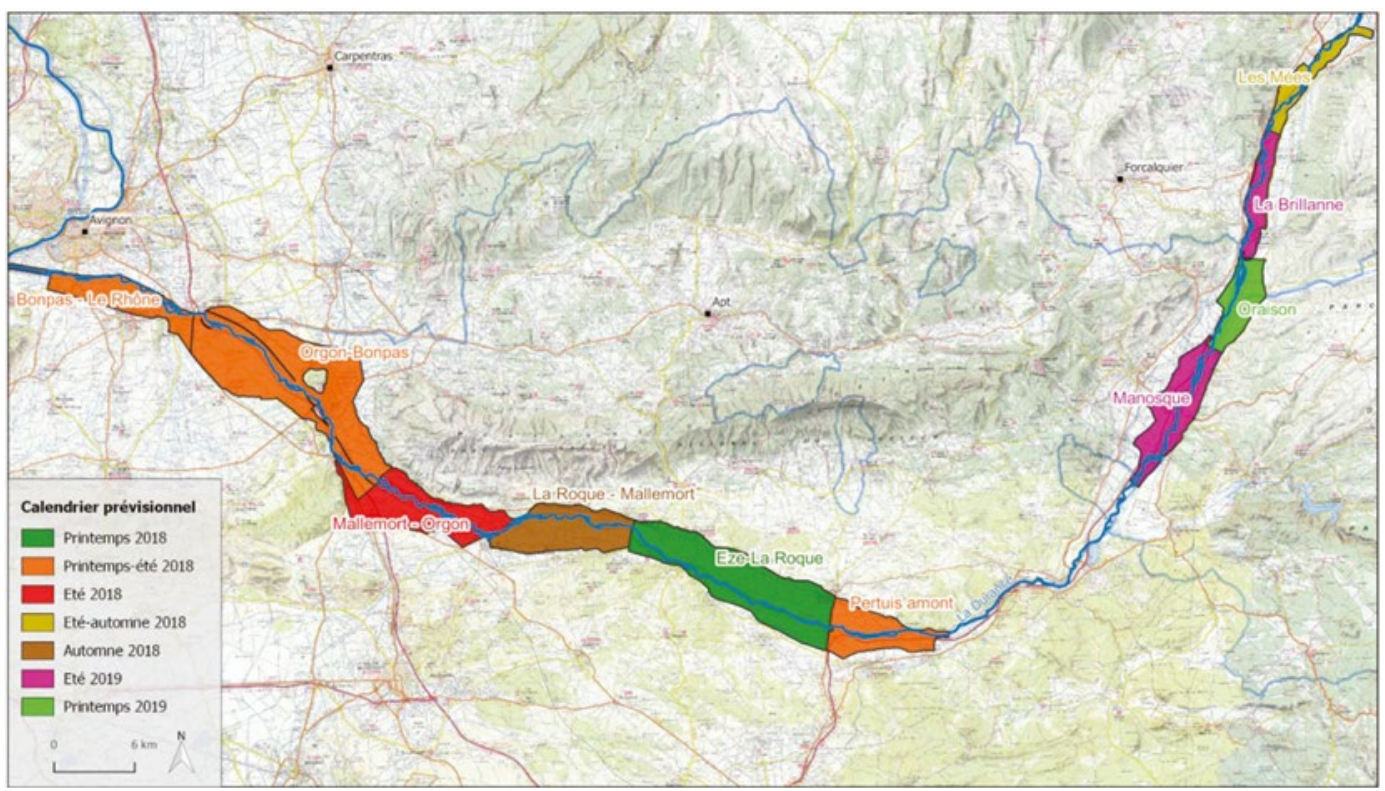

Figure 6 : Emprise de déploiement des Atlas Dynamique 2018-2019.

En période normale, l'outil permet de disposer de l'ensemble des données hydrauliques nécessaires à une préparation efficace aux futures crises. On retiendra par exemple :

- La mise au point d'un plan communal ou intercommunal de sauvegarde (Communes et Intercommunalités),

- La définition d'une stratégie de fermeture / déviation d'axes routiers sensibles au risque d'inondation,

- La préparation de procédures de gestion des réseaux en période de crue (GRT Gaz, RTE, Enedis, France Telecom...), - L'organisation d'exercices préventifs de mise en situation de crue...
En phase de réflexion sur la mise en œuvre de la GEMAPI dans des territoires fortement équipés en ouvrages peu fiables et complexes, l'outil éclaire sur les enjeux exposés dès les premières crues débordantes et permet de définir une stratégie politique de défense contre les inondations.

En période de crise, l'outil permet avant tout à chacun des acteurs de se projeter de manière opérationnelle pour ce qui le concerne et de structurer ses prises de décisions propres. La mise en page unifiée, la sémiologie graphique partagée et la mise à jour régulière conduit par ailleurs à placer l'atlas dynamique des zones inondables comme un outil facilitant la communication et la coordination. 


\section{CONCLUSION : DES PERSPECTIVES D'INTÉRACTIVITÉ}

Les résultats de modélisation permettent désormais un rendu dynamique de l'évolution des zones inondables avec l'évolution progressive des débits. Le travail d'intégration des scénarios de tenue/rupture des ouvrages a solutionné le problème posé par l'ensemble des acteurs de la gestion du risque en matière de préparation comme de gestion de la crise. Les productions seront intégrées au fil de l'eau dans la plateforme nationale ZIP.

Les nouvelles technologies doivent permettre à l'avenir d'aller au-delà des classiques représentations en plan afin d'offrir à l'utilisateur averti une expérience plus immersive et à de nouvelles cibles d'initier ou de compléter leur culture du risque inondation.

Il est ainsi envisagé de développer une application mobile, dont les principales fonctions pourraient être :
- De disposer des messages de prévention ou d'alerte en fonction de la localisation du terminal

- De consulter les cartes en «web SIG»

- De visualiser les inondations potentielles en réalité augmentée en certains points remarquables

- D'interagir en faisant remonter des constats de terrain...

\section{RÉFÉRENCES}

Doddoli C., Moreau L., Picon P. Pignoly H. (2015) - Contrat de Rivière du Val de Durance, Avenant au contrat initial. 134-135.

Jousse C., JACOPIN B. (2015) - Restructuration des dispositifs de protections contre les crues entre Pertuis et Villelaure. 73.

Mercier F., Jacopin B. (2017) - Restructuration des dispositifs de protections contre les crues entre Pertuis et Villelaure, Mise à jour de l'étude hydraulique suite à la crue de novembre 2016 . 3-6. 